\title{
ESTIMATIVA DA PREVALÊNCIA DE NEOPLASIA CEREBRAL NA FAIXA ETÁRIA PEDIÁTRICA PELO MÉTODO DE CAPTURA-RECAPTURA
}

\author{
NAYARA ARGOLLO*, INES LESSA **
}

\begin{abstract}
RESUMO - O estudo teve como objetivo estimar a prevalência e a letalidade das neoplasias cerebrais primárias em crianças entre 1-15 anos, Bahia, 1995. O desenho foi de corte transversal, incluindo todos os casos de neoplasia cerebral diagnosticados por múltiplas fontes. A partir dos casos identificados foi utilizado o método de capturarecaptura para estimar o provável número real de casos e medir as prevalências, com intervalos de confiança (IC) a 95\%. Por este método estimou-se a existência de 87 casos $(\mathrm{IC}=64 ; 111)$ de neoplasias cerebrais no Estado, sendo 72 (IC =54 ; 91) na capital. A prevalência resultante foi 1,85/100 000 crianças entre 1 e 15 anos (IC =1,36; 2,36/100 000) para o Estado e 10,1/100 000 (IC = 7,58;12,77/100 000) para a capital. A letalidade foi 5,4\% (IC $=4 \% ; 8 \%)$ para a capital e $8,9 \%$ (IC $=7 \% ; 13 \%)$ para o estado. O método de captura-recaptura mostrou elevada precisão na estimativa do total de casos. Possíveis causas para o não diagnóstico e/ou tratamento das neoplasias cerebrais são comentadas.
\end{abstract}

PALAVRAS-CHAVE: neoplasias cerebrais primárias, prevalência, letalidade, captura-recaptura.

\section{Estimative prevalence of cerebral neoplasm of childhood from the capture-recapture method}

ABSTRACT- The aim of the study was to estimate the prevalence and fatality rate of primary cerebral neoplasms among children between 1 and 15 years old, State of Bahia, Brazil,1995. It was a cross-sectional study which includes all cases of brain tumors diagnosed by several sources. From these cases the capture-recapture method was used to estimate the probable number of cases and measure the prevalence and confidence intervals at $95 \%$. By this method it was estimated the existence of 87 cases $(C I=64 ; 111)$ of brain tumores in the State, being 72 $(\mathrm{CI}=54 ; 91)$ in the capital. The estimated prevalence was $1.85 / 100000$ for children between 1 to 15 years old $(\mathrm{CI}=1.36 ; 2.36 / 100000)$ for the State, and $10.1 / 100,000(\mathrm{CI}=7.58 ; 12.77 / 100,000)$ for the capital. The fatality rate was 5.4 percent $(\mathrm{CI}=4 \% ; 8 \%)$, and $8.9 \%(\mathrm{CI}=7 \% ; 13 \%)$ for the capital and the State respectively. The capture-recapture method showed high precision to estimate the total number of cases. Possible causes of no diagnostic nor treatment of cerebral neoplasms are discussed.

KEY WORDS: primary cerebral neoplasms, prevalence, fatality rate, capture-recapture method.

Dentre todas as neoplasias, $1,5 \%$ ocorre em crianças, porém, $15 \%$ a $20 \%$ de todos os tumores intracranianos ocorre na infância. Além disso, $19 \%$ de todas as neoplasias em pacientes menores de 15 anos envolve o sistema nervoso central (SNC) $)^{1,2}$. São o segundo maior grupo de neoplasias na infância, perdendo somente para as leucemias ${ }^{3}$, constituindo-se, nesta faixa, no maior grupo de tumores sólidos ${ }^{1,4}$. Têm maior probabilidade de levar à morte $(45 \%)$ do que o conjunto de todas as

Faculdade de Medicina da Universidade Federal da Bahia (UFBA); * Mestre em Assistência MaternoInfantil, Pediatra/Neuropediatra; ** Doutor em Medicina, Mestre em Saúde Comunitária, Pesquisador IA do CNPQ, Professor dos cursos de pós-graduação do Instituto de Saúde Coletiva da UFBA. Aceite: 10-março-1999.

Dra. Nayara Vieira - Av. Tancredo Neves 805 A sala 103 - 41820-023 Salvador Bahia - Brasil. Fax 071358 0972. E-mail: rnvieira@svn.com.br 
leucemias $(42 \%)^{1,5,6}$ representando a terceira causa de óbito nos Estados Unidos ${ }^{7}$. Apesar da baixa incidência (variação entre 1,5 a 4 casos por 100000 crianças abaixo de 15 anos) ) $^{8-10}$, as neoplasias cerebrais representam patologias clinicamente importantes pela sua gravidade e pela dificuldade do diagnóstico e tratamento precoces ${ }^{11}$. O diagnóstico requer exames de alta tecnologia, e tratamento de elevada complexidade envolvendo equipe médica multidisciplinar ${ }^{11}$.

No Brasil são raros os estudos sobre neoplasias cerebrais na infância, sobretudo os de natureza populacional, que dimensionam o problema para programações de ações em saúde. Com base nos dados das cidades de Belém (1988 e 1989), Fortaleza (1985), Campinas (1991 e 1992), Porto Alegre (1990 e 1991) e Goiânia (1990 e 1991), foi possível calcular uma incidência de 1,89 por 100000 na população de 0 a 14 anos, para o Brasil em 1995 ${ }^{12}$. Os dados procedentes do único serviço de oncologia pediátrica do Estado da Bahia, até 1984, situavam o tumor cerebral no $9^{\circ}$ lugar $(2,5 \%$ do total), suspeitando-se a baixa frequência diagnóstica pelo não encaminhamento dos casos para tratamento e acompanhamento em serviço de oncologia ${ }^{13}$.

Este representa parte de um estudo maior sobre neoplasias cerebrais na infância no Estado da Bahia e o objetivo consiste em estimar a prevalência e letalidade na população de 1 a 15 anos, utilizando o método da captura-recaptura, propiciando os elementos necessários para o planejamento em saúde.

\section{MÉTODO}

Estudo realizado utilizando toda a casuística de tumores cerebrais entre 1 e 15 anos identificada em todos os serviços de diagnóstico e os especializados em tratamento, existentes no Estado da Bahia no primeiro semestre de 1995. Os pacientes tinham até 5 anos de diagnóstico e tratamento, uma vez que este é o período de acompanhamento dos sobreviventes até a alta.

Para a medida da prevalência, o estudo foi de corte transversal, utilizando-se no numerador o número de casos de tumores cerebrais previamente estimado pelo método da captura-recaptura, com base na casuística total obtida de cada fonte de dados. Este número também foi utilizado como denominador para a determinação da taxa de letalidade no mesmo período.

As fontes de dados utilizadas foram:

Fonte 1 - Corresponde às "fontes diagnósticas" e inclui:

a) Revisão dos laudos dos exames de tomografia computadorizada (TC) e ressonância magnética (RM) do crânio de todos os serviços de neuroimagem existentes no Estado. Salvador dispunha em 1995 de 2 serviços com aparelhos de RM, um deles conveniado ao Sistema Único de Saúde (SUS), mas que só realizava exames, para este, em situações especiais, mediante autorização da Secretaria de Saúde; 10 serviços com TC (8 em hospitais, 4 dos quais atendiam também pelo SUS e 2 em clínicas privadas, conveniadas ao SUS). Dos oito hospitais, um era federal e um estadual (excluído do trabalho por não haver registro dos laudos); no interior havia um serviço de TC em cada uma das cidades: Itabuna, Ilhéus, Vitória da Conquista, Feira de Santana e Juazeiro. Todos pertenciam a clínicas ou hospitais particulares, mas conveniados com o SUS. Na cidade de Vitória da Conquista não havia arquivo dos laudos, e na cidade de Juazeiro não havia registro da idade dos pacientes. Ambas as cidades foram excluídas da fonte diagnóstica. Em todos os demais serviços foram separados os laudos com diagnóstico de massa expansiva. Nos casos cujos laudos não eram conclusivos para neoplasia cerebral, foram contatados por telefone os familiares e/ou médicos dos pacientes para confirmação; na impossibilidade do esclarecimento, o caso era excluído.

b) Revisão dos laudos de neoplasia cerebral em todos os laboratórios de anatomia patológica existentes no Estado: 14 em Salvador (4 estaduais, 1 federal e 9 particulares) e um em cada uma das cidades anteriormente mencionadas. Nestas cidades, todos os laboratórios eram particulares, conveniados ao SUS.

Fonte 2 - Corresponde às "fontes de tratamento" e inclui:

a) Revisão dos prontuários dos 3 serviços de oncologia pediátrica de Salvador e um na cidade de Itabuna.

b) Revisão dos prontuários de pacientes com neoplasia cerebral de todos os serviços de radioterapia: dos 5 serviços existentes, 4 localizados na capital e um em Itabuna.

Das fontes diagnósticas e de tratamento foram anotadas: idade, sexo, endereço, telefone, médico solicitante, data do diagnóstico, diagnóstico e fonte pagadora do exame. 
Tabela 1. Distribuição dos pacientes segundo as fontes de identificação para o cálculo do ODDs ratio e verificação de dependência positiva ou negativa.

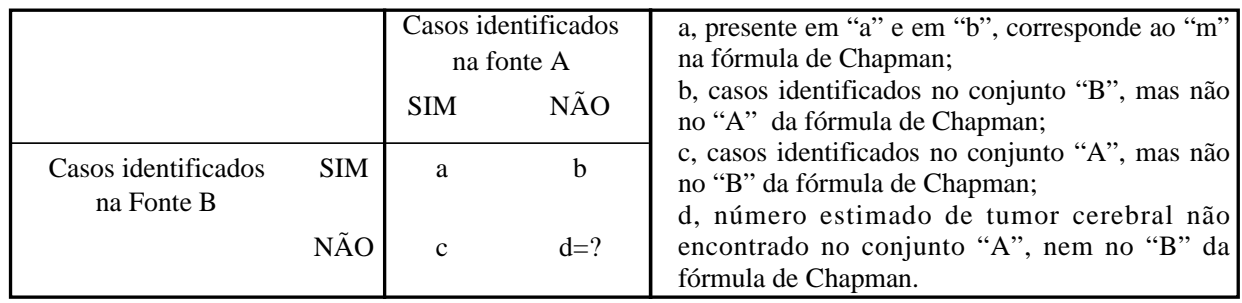

Obs: A estimativa de d= bc/a, sendo 'd' correspondente a estimativa do número de casos que não foram achados em nenhuma das fontes. Obtido o 'd', a fórmula para o cálculo do OR é a mesma dos estudos caso-controle não pareado: $\mathrm{OR}=\mathrm{ad} / \mathrm{bc}$. $\mathrm{A}$ população total (casuística) - $\mathrm{N}$ - (com correção para amostras pequenas) é: $\mathrm{N}=\mathrm{a}+\mathrm{b}+\mathrm{c}+\mathrm{bc} / \mathrm{a}+1$

Fonte 3 - Declaração de óbito - Todas as declarações de óbitos de crianças entre 1 e 15 anos de idade foram revistas no Centro de Informação em Saúde do Estado da Bahia e separados aqueles em que a causa declarada da morte foi tumor cerebral. Como a informatização excluiu o nome das pessoas falecidas, não foi possível cruzar os casos com os das outras fontes. Por essa razão esta fonte não foi computada na análise do método de captura-recaptura, sendo apenas utilizada para os cálculos de letalidade, segundo local de procedência do caso.

Também foram excluídos do estudo os tumores metastáticos e malformativos, exceto o craniofaringioma, pelo seu comportamento maligno.

Foi utilizado o método de captura-recaptura e seus intervalos de confiança (IC) a 95\% para estimar o provável número de casos realmente existente no Estado. Este método baseia-se no cálculo do grau de sobreposição entre duas ou mais fontes de dados para estimar o provável número dos casos de determinada patologia em uma população ${ }^{14}$. Para tal, utiliza-se a fórmula de Chapman, quando se usam duas fontes de dados. O fato de não serem as amostras compostas por todos os indivíduos atingidos não preocupa, uma vez que as estimativas são feitas com base no número de pacientes que aparecem nas duas amostras, ou seja, a estimativa é feita sobre o grau de concomitância dos $\operatorname{casos}^{14,15}$. O método é simples e acurado ${ }^{15}$, possibilitando a estimativa do número de casos e a determinação do "odds ratio", e verificação de dependência positiva ou negativa das fontes. Por tratar-se de método antigo, mas só recentemente introduzido na investigação médica, foram incluídos os passos necessários à estimativa do número de casos de neoplasia cerebral em cada fonte. A Figura 1 mostra como se dá a superposição de duas fontes, acompanhada da fórmula de Chapman para as estimativa pretendidas; a Tabela 1 demonstra o procedimento para o cálculo do "odds ratio" usando as duas fontes de dados.

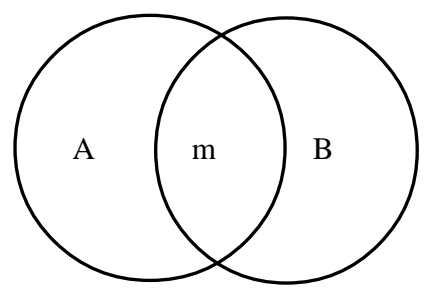

Fórmula de Chapman (LaPorte, 1993)

$$
\begin{aligned}
& \mathrm{N}=\text { população total } \\
& \mathrm{A}=\text { Primeira amostra } \\
& \mathrm{B}=\text { Segunda amostra } \\
& \mathrm{m}=\text { Indivíduos em ambas as amostras } \\
& \mathrm{Ne}=\frac{(\mathrm{A}+1)(\mathrm{B}+1)}{\mathrm{m}+1}-1
\end{aligned}
$$

Ne é igual à estimativa da população total (no caso do presente estudo, casuística).

$$
\text { Variância }(\mathrm{Ne})=\frac{(\mathrm{A}+1)(\mathrm{B}+1)(\mathrm{A}-\mathrm{m})(\mathrm{B}-\mathrm{m})}{(\mathrm{m}+1)(\mathrm{m}+1)(\mathrm{m}+2)} \quad 95 \% \text { Intervalo de confiança } \mathrm{CI}= \pm 1,96 \sqrt{\operatorname{Var}(\mathrm{Ne})}
$$

Fig 1. Superposição de duas fontes de dados pelo método da captura-recaptura. 
Figura 2.1

Neuroimagem

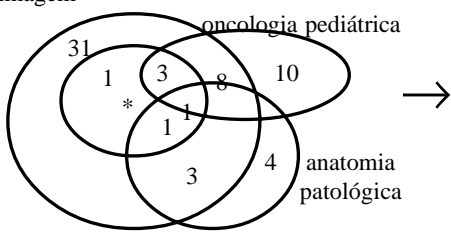

*radioterapia
Figura 2.2

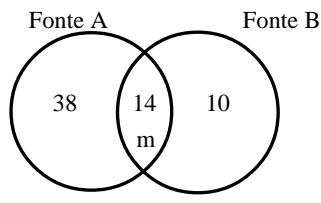

Fonte $\mathrm{A}=$ neuroimagem e anatomia patológica

Fonte $\mathrm{B}=$ oncologia pediátrica e radioterapia $\mathrm{m}=$ casos comuns às duas fontes

Fig 2. Fontes de diagnóstico e de tratamento do Estado (2.1) e seus agrupamentos (2.2)

A partir dos casos estimados foram determinados:

a) a prevalência para o Estado e capital, em que o numerador correspondeu aos casos estimados pelo método da captura-recaptura e o denominador, a população de crianças de 1-15 anos do Estado da Bahia e da capital, separadamente, no mesmo período de tempo;

b) a letalidade para o Estado e capital, foi calculada utilizando no numerador os óbitos levantados nas declarações de óbitos e no denominador o número de casos prevalentes estimado.

\section{RESULTADOS}

Foram encontrados 52 casos nas fontes diagnósticas e 24 nas de tratamento, havendo 14 casos em comum. Para a capital, 43 casos na fonte diagnóstica e 24 nas de tratamento e 14 casos em comum. As Figuras 2.1 e 3.1 representam todas as fontes de dados do Estado e da capital e a interseção de todas as fontes são transformadas em apenas duas que aparecem na Figura 2.2 e 3.2.

$\mathrm{Na}$ avaliação da dependência entre as fontes, o "odds ratio" foi igual a 1, demonstrando não haver dependência elas. Pela fórmula de Chapman foi encontrado que no Estado o "Ne" foi 87,3 casos de

\section{Figura 3.1}

Neuroimagem

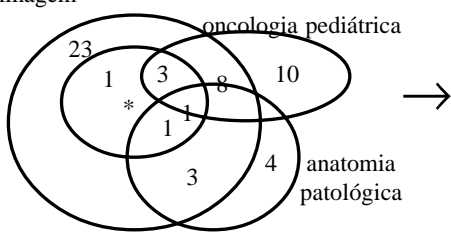

*radioterapia
Figura 3.2

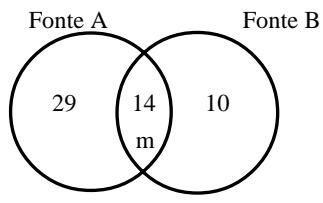

Fonte $\mathrm{A}=$ neuroimagem e anatomia patológica Fonte $\mathrm{B}=$ oncologia pediátrica e radioterapia $\mathrm{m}=$ casos comuns às duas fontes

Fig 3. Fontes de diagnóstico e de tratamento da capital (3.1) e seus agrupamentos (3.2). 
Tabela 2. Grau de acerto das fontes A e B pelo método de captura-recaptura no Estado da Bahia.

\begin{tabular}{|c|c|c|c|c|c|c|}
\hline & Fonte A & Fonte B & Casos comuns & Identificados & Estimados & IC $95 \%$ \\
\hline Número de casos & 52 & 24 & 14 & 62 & 87 & $(64-110)$ \\
\hline Grau de acerto $(\%)$ & 59,8 & 27,6 & & 71,3 & & \\
\hline $\begin{array}{l}\text { Intervalo de confiança } \\
(95 \%) \text { do grau de acerto }\end{array}$ & $81,3-47,3$ & $37,5-21,8$ & & $96,9-56,4$ & & \\
\hline
\end{tabular}

neoplasia cerebral, IC a 95\% = 64,1; 110,5. Na capital o "Ne" de 72,3 casos, com IC a 95\% = 53,9; 90,8.

A prevalência de tumor cerebral encontrada para o Estado foi $1,85(\mathrm{IC}=1,36 ; 2,36)$ por 100000 crianças $1-15$ anos e para a capital o valor foi 10,1 (IC $=7,58 ; 12,77)$.

Oito óbitos ocorreram no primeiro semestre de 1995: 4 na capital e 4 no interior. Destes, 7 foram no sexo feminino, sendo 5 na faixa etária de 5 a 9 anos. A letalidade no Estado foi de $8,9 \%$ (IC $=6,9 \% ; 12,5 \%)$. Na capital a letalidade foi de $5,4 \%$ (IC $=4,2 \% ; 7,6 \%)$.

As Tabelas 2 e 3 demonstram o grau de acerto das fontes A e B no Estado da Bahia e na capital pelo método de captura-recaptura. Para a fonte A, o grau de acerto foi superior ao da fonte B, e semelhante tanto para a capital como para todo o Estado, mas o grau de acerto para os casos identificados foi superior na capital.

\section{DISCUSSÃO}

A falta de registros de câncer dificulta a realização de um estudo de incidência de neoplasia cerebral no Estado da Bahia. Se, por um lado, coletar esta informação diretamente da população não seria o método de escolha para doenças raras como esta, por outro, o levantamento exaustivo nas diversas fontes diagnósticas e de tratamento não cobriria todos os casos. Isto pode ser de imediato comprovado pela discrepância entre os diagnosticados nesta casuística e aqueles em tratamento. $\mathrm{O}$ método da captura-recaptura reduziu esta deficiência, permitindo estimar com precisão (pequena variação dos intervalos de confiança em relação ao valor central estimado) os possíveis casos existentes, porém não registrados, quer nas fontes diagnósticas, quer nas de tratamento. O método presume que, em se tratando de doença rara, a perda de poucos casos - como talvez tenha ocorrido nas duas cidades sem condição do levantamento de dados ou nos hospitais excluídos por questões das suas deficiências - não prejudica a estimativa, uma vez que a chance de recapturar os casos perdidos, nas múltiplas fontes, é muito grande.

Vale ressaltar que o hospital estadual excluído do estudo é o principal e único hospital exclusivo para urgências e emergências médicas do Estado, e ao seu pronto socorro são encaminhados casos de tumor cerebral, geralmente em fase tardia, com sintomatologia grave, como a hipertensão intracraniana e em risco de vida. Estas crianças poderiam ser recapturadas em outros serviços, como o de anatomia

Tabela 3. Grau de acerto das fontes A e B pelo método de captura-recaptura na cidade de Salvador.

\begin{tabular}{lccccc}
\hline & Fonte A & Fonte B & Casos Comuns & Identificados & Estimados IC 95\% \\
\hline Número de casos & 43 & 24 & 14 & 53 & 72 \\
Grau de acerto (\%) & 59,7 & 33,3 & & 73,6 & \\
Intervalo de confiança & $79,6-47,3$ & $44,4-26,4$ & & $98,1-58,2$ & \\
$(95 \%)$ do grau de acerto & & & & \\
\hline
\end{tabular}


patológica, para onde são remetidas as peças cirúrgicas deste hospital ou nos serviços de oncologia pediátrica ou radioterapia. Se transferidas para outro hospital seriam recapturadas nos exames de controle de tomografia pós-cirúrgicos ou nos laboratórios de anatomia patológica ou ainda recapturadas através dos certificados de óbito. Somente os pacientes que faleceram sem tratamento seriam perdidos, ou aqueles transferidos para outro Estado. Estas duas possibilidades representam situações raras, pois este hospital atende predominantemente pacientes do SUS sem possibilidade de transferência para outro Estado e nenhum dos 4 atestados de óbito da capital teve procedência deste hospital. Portanto, não ocorreu número de casos perdidos por óbito neste hospital, exceto se na declaração de óbito constou apenas diagnóstico sintomático, situação em que qualquer doença é classificada como "causa mal definida de morte". Essa é uma possibilidade explicativa para o reduzido número de óbitos.

Nenhuma fonte de dados contempla o número total de casos existentes de determinadas doenças, sobretudo quando o diagnóstico é de difícil acesso pelos estratos sociais mais baixos, como é o caso das neoplasias cerebrais na infância. As listas de casos coletados nas diversas fontes eram, portanto, incompletas e a simples somatória do número de casos encontrados não corresponderia ao verdadeiro número de casos de neoplasia no Estado da Bahia, por isso foi utilizado o método de captura-recaptura. As fontes escolhidas para o método de captura-recaptura demonstraram ter bom grau de acerto (>70\%) quando foram comparadas com os casos estimados pelo método; portanto, foram consideradas como fontes representativas do total de casos.

A grande diferença entre as prevalências de tumor cerebral no Estado (1,85/100 000) e capital $(10,1 / 100.000)$ sugere que o número de casos diagnosticados na capital está superestimado pela inclusão de casos procedentes do interior, onde não se dispunha, até 1995, de nenhum serviço de oncologia pediátrica para tratamento quimioterápico. Consequentemente, a taxa de prevalência do interior estaria subestimada ou então o diagnóstico não estaria sendo realizado, em razão do difícil acesso à assistência médica, aos meios diagnósticos e de tratamento, ou ainda pela má qualidade assistêncial.

Apenas um caso de tumor foi encontrado nos serviços de anatomia patológica nas cidades do interior; portanto, pacientes pediátricos com tumor cerebral não estão sendo tratados cirurgicamente nestas cidades. Em Itabuna, a única que tem serviço de radioterapia, não foi encontrado caso de criança com neoplasia cerebral em tratamento com radioterapia.

A letalidade encontrada de 8,9\% para o Estado e 5,4\% para a capital, não pode ser comparada a outros estudos pela falta de dados equivalentes na literatura. Deve ser ressaltado, contudo, que as causas mal definidas de óbito têm alta frequência no Estado da Bahia, especialmente nas cidades do interior (variação entre 27 e 97) além do que, para estas causas, a morte ocorre sem assistência médica entre 25 e $100 \%$ dos casos (Lessa I, comunicação pessoal, 1996). Portanto, é provável que os casos obtidos pelas declarações estejam, de fato, subestimados. Chama atenção a constatação de 19 casos novos no Estado e número pequeno de casos nos serviços de anatomia patológica, radioterapia e oncologia pediátrica, o que leva a supor que, as crianças com o diagnóstico confirmado de neoplasia cerebral, não estejam sendo tratadas cirurgicamente nem com quimioterapia ou radioterapia.

\section{CONCLUSÕES}

As neoplasias cerebrais na infância são de baixa prevalência populacional, mas clinicamente importantes, podendo deixar sequelas graves e levar ao óbito. Este estudo demonstra concentração de casos na capital, sinalizando para um baixo grau de suspeita clínica e diagnóstico no interior do Estado. Poucos pacientes estão tendo acesso à cirurgia para a remoção do tumor, como mostra a baixa recaptura dos paciente nos serviços da anatomia patológica, o mesmo ocorrendo nos serviços de oncologia pediátrica e radioterapia. É possível que subestimativas de diagnósticos, no interior do Estado, sejam decorrentes da falta de diagnóstico e de assistência médica, levando à morte por "causa mal definida", fato comum no interior do Estado. O mesmo raciocínio aplica-se ao pequeno número de óbitos por tumor cerebral. 
O método de captura-recaptura, embora possa apresentar tendenciosidades quando as fontes utilizadas não são bem exploradas, mostrou precisão neste estudo, detectada pela pequena variação dos intervalos de confiança a $95 \%$. Portanto, considera-se que o resultado da prevalência populacional é confiável.

\section{REFERÊNCIAS}

1. Lannering B, Marky I, Nordborg C. Brain tumors in childhood and adolescence in West Sweden, 1970-1984: epidemiology and survival. Cancer1990;66:604-609.

2. Marold TV, Barkovich AJ. Pediatric brain tumors. Seminars in Ultrassound, CT, and MRI, 1992;13:412-448.

3. Miller RW, Young JL, Novakovic B. Childhood cancer. Cancer 1995;75:395-405.

4. Pollack JF. Brain tumors in children. N Engl J Med, 1994;329:1500-1507.

5. Duffner PK, Horowitz ME, Krisher JP. Potoperative chemotherapy and delayed radiation in children less than three years of age with malignant brain tumors. N Engl J Med, 1993;328:1725-1731.

6. YoungJ, Miller R. Incidence of malignant tumors in US children. J Pediatr, 1975;86:254-258.

7. Shiminski-Maher T, Wisoff JH. Pediatric brain tumors. Crit Care Nursing Clin N Am,1995;7:159-169.

8. Drut R, Hernández A, Pollono D. Incidende of childhood cancer in La Plata, Argentina, 1977-1987. Int J Cancer 1990:45:1045-1047.

9. Schoenberg BS, Schoenberg DG, Christine BW. The epidemiology of primary intracranial neoplasms of childhood: a population study. Mayo Clin Proc 1976;51:51-56.

10. Young SL, Ries LG, Silverberg E, Horn JW, Miller RW. Cancer incidence, survival and mortality for children younger than 15 years. Cancer 1986;58:598-602.

11. Baron Mc. Advances in care of children with brain tumors. J Neurosc Nursing 1991;23:39-43.

12. Ministério da Saúde. Instituto Nacional de Câncer: Câncer no Brasil: dados dos registros de base populacional, Volume II, 1995.

13. Mendonça N. Tumores cerebrais. In Mendonça N (ed): Manual diagnóstico do câncer na criança. Salvador: Bureau Gráfica e Editora,1984.

14. MacCarter D, Kwoh C, La Porte R. The importance of incidence registries for connective tissue diseases. J Rheum, 1992;1:1-7.

15. La Porte R. Assessing the human condition: capture-recapture techniques. BMJ 1994;308:5-6. 